\title{
Autotransplantation of an Impacted Second Premolar in its Predecessor's Socket with the Novel use of Modern Imaging System: A Case Report with 18 Months Follow Up
}

\author{
Divya S Sharma ${ }^{1 *}$, Shikhar Pratap Singh Chauhan', Rajesh Kishnan ${ }^{2}$ and Sanjay Sharma ${ }^{3}$
}

${ }^{1}$ Department of Pediatric and Preventive Dentistry, Modern Dental College \& Research Centre, Indore (MP), India

${ }^{2}$ Department of Orthodontics, Modern Dental College \& Research Centre, Indore (MP), India

${ }^{3}$ Department of Prosthodontics, Crown \& Bridge and Implantology, Modern Dental College \& Research Centre, Indore (MP), India

\begin{abstract}
The case repost presents a successful autotransplantation of maxillary second premolar, impacted at an unusual site, in its predecessor's socket. The location of impacted tooth was diagnosed with the help of 3D construction 3D CT scan. 3D construction was further modified to unveil the exact location of impacted tooth making best use of softwares incorporated in modern imaging systems. The good diagnosis decreased the time to be taken in surgical procedure which in turn resulted in successful outcome of autotransplantation.
\end{abstract}

Keywords: Impaction; Autotransplantation; 3d Image construction

\section{Introduction}

Autotransplantation is considered a useful method for replacing missing anterior or posterior teeth with transplanted autogenous donor tooth. The procedure has been indicated for various clinical conditions including aplasia, displacement, caries/osteitis, trauma, infraocclusion, periodontitis etc [1-3]. Autotransplantation has been considered a better option than any prosthetic option in growing individuals as not only it helps in growth but also preserves alveolar bone three dimensionally. Also there is possible avoidance of space maintainers during interim period till prosthetic placement [4].

Labio/buccal - lingual/palatal impactions often pose difficulty for orthodontic movement which needs controlled movement probably in all directions. Orthodontic traction may require to wait till the root completion of impacted tooth in order to avoid complications viz. dilacerations, non-vitality, root resorption etc. On the other hand if impacted tooth is allowed to grow in crowded arch, may result in bizarre root anatomy, making future orthodontic treatment difficult if not impossible. Also post retention period for an orthodontically moved tooth is increased. Therefore autotransplantation of developing impacted tooth into its proper position seems to be a best treatment option.

The case report presents successful autotransplantation of an impacted developing maxillary left second premolar in the socket of over-retained maxillary left deciduous second molar, its predecessor, with 18 months follow up.

\section{Case Report}

The impaction of developing maxillary left second premolar in a 10 year boy was detected in routine orthopentogram (OPG) being done at regular intervals for serial extraction (Figure 1). Patient did not complain of any discomfort. Intra-oral examination revealed all first premolar extracted and all permanent teeth were erupting well except for all retained second deciduous molars. A prominent bulge on the left palate in primary maxillary left second molar region was seen (Figure 2). The bulge was hard, non-tender and non-inflammatory, indicating palatal impaction of maxillary left second premolar. Nonetheless, exact angulations of impaction could not be determined with OPG. Hence CT scanning was planned.
Prior permission from local hospital committee and informed consent from parents was obtained. 3-D CT scanning was performed on Siemens multidetector CT scanning station with the following parameters: $120 \mathrm{kVp}, 200 \mathrm{~mA}$, rotation time of $1.0 \mathrm{sec}$., pitch 1.8, slice thickness of $1 \mathrm{~mm}$ and HQ mode (Figure $3 \mathrm{~A}$ ). All these sectional images gave idea that impacted tooth was located palatally with crown directed mesially and whole tooth was lying apical to the roots of primary maxillary left second molar. Root images (except for tip of palatal one) of primary maxillary left second molar were not seen in the axial view of head at impaction level indicative of impaction lying apical to roots of its predecessor (Figure 3B).

Visualization of impaction was further improved with 3D construction of images using raw data of sectional CT images. To select the relevant structures (i.e. teeth) the window width was adjusted

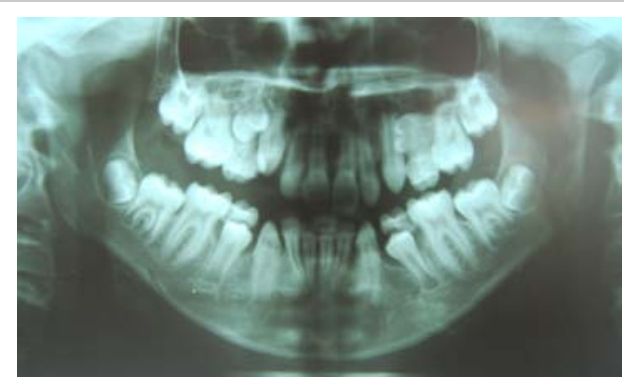

Figure 1: Impacted 25 detected in Routine Opg.

*Corresponding author: Divya S Sharma, Department of Pediatric and Preventive Dentistry, Modern Dental College \& Research Centre, Indore (MP), India, Tel: +919977701098; E-mail: drdivyassharma@gmail.com

Received November 24, 2011; Accepted December 27, 2011; Published January 10, 2012

Citation: Sharma DS, Singh Chauhan SP, Kishnan R, Sharma S (2012) Autotransplantation of an Impacted Second Premolar in its Predecessor's Socket with the Novel use of Modern Imaging System: A Case Report with 18 Months Follow Up. J Trauma Treat 1:103. doi:10.4172/2167-1222.1000103

Copyright: (c) 2012 Sharma DS, et al. This is an open-access article distributed under the terms of the Creative Commons Attribution License, which permits unrestricted use, distribution, and reproduction in any medium, provided the original author and source are credited. 
Citation: Sharma DS, Singh Chauhan SP, Kishnan R, Sharma S (2012) Autotransplantation of an Impacted Second Premolar in its Predecessor's Socket with the Novel use of Modern Imaging System: A Case Report with 18 Months Follow Up. J Trauma Treat 1:103. doi:10.4172/21671222.1000103

according to the Hounsfield gray scale. On this scale, a value of - 1024 is assigned to air, 0 is assigned to water. Trabecular bone is in the range of 100 to 300 and teeth extend above trabecular bone. So only the tissues with higher densities were selected and those with lower densities were excluded. This revealed all the teeth lying within the bone also. Color coding (pink) was assigned using the VRT (Volume Rendered Technique) (Figure 4A). The entire endeavor was done to unveil the impacted maxillary left second premolar. This revealed bucco-palatal impaction of maxillary left second premolar in the space between primary maxillary left second molar and permanent maxillary left first molar with the crown on mesio-palatal side and root bucco-posteriorly between the roots of primary maxillary left second molar and maxillary left first molar.

\section{Treatment}

The case was discussed with orthodontist for whether it can be brought into occlusion or not. The case was further discussed with prosthodontist for two possibilities 1) extra-coronal restoration of maxillary left second premolar with dimensions of second premolar, 2) growth and preservation of alveolar bone till implant is scheduled. According to prosthodontist both options were open and to be decided as case progress. Tentative treatment plan of extraction of primary maxillary left second molar, palatal exposer of crown of maxillary right second premolar followed by button attachment on buccal surface was made. It was expected that light force would rotate the teeth moving its root palatally and crown buccally in the extracted space of primary maxillary left second molar. On scheduled date after blood investigation and prophylactic antibiotic regime, surgery was started in the department of Pediatric dentistry, Modern Dental College, Indore (India).

Buccal infiltration on left side and greater palatine nerve block on both side was given. Release incison was made distal to the primary maxillary left second molar. Palatal full thickness flap was raised high till the crown of premolar was visible (Figure 5). Its position was such that it was orthodontically impossible to bring into occlusion. As 3-D CT construction showed only two third of root completion of canine (right and left) and second premolar (right side), same status of root development of maxillary left second premolar was assumed (Figure 4B). Immediately the treatment plan was changed. Treatment plan of extraction of primary maxillary left second molar followed by disimpaction of maxillary left second premolar and autotransplantation of it into the socket of extracted tooth was made. Written consent from parent was obtained for changed treatment plan. Primary maxillary left second molar was extracted with vertical subluxation with the help of straight elevator followed by forceps. Roots of primary maxillary left second molar were in resorbed state. Maxillary left second premolar

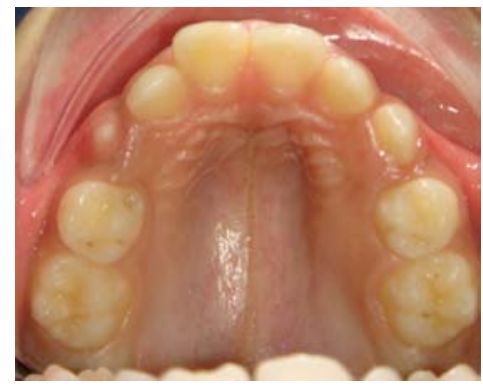

Figure 2: Palatal bulge, indicating impaction on Palatal Side.

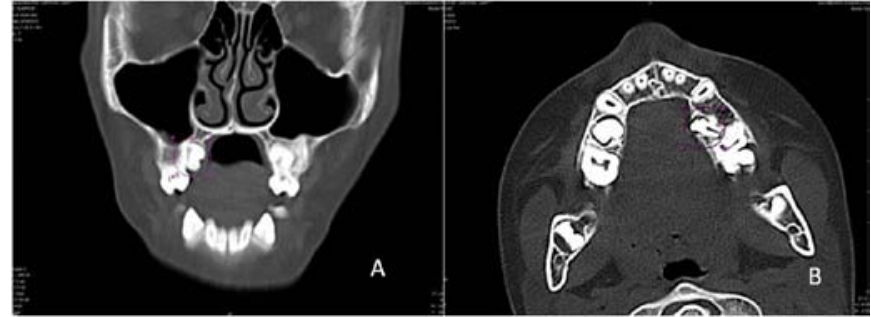

Figure 3: A. Coronal view of PNS ( 3 X magnification) showing crown of impacted 25 (encircled) on palatal side. B. The axial view of head ( $3 \times$ magnification) showing crown and root of 25 with crown located mesio-palatally to its root and whole tooth located apical to the roots of 65 . Palatal bone perforation by crown of 25 is evident. It was difficult to imagine with this image that whether 25 had single root and open apex, or it had two roots.

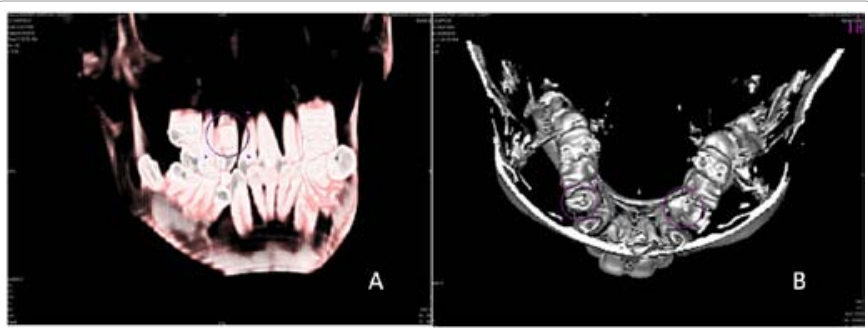

Figure 4: A. 3-D construction of maxillary jaw. Colour coding was assigned using the Volume Rendered Technique. Encircled image shows the impacted 25 with the crown directed palatally. B. 3-D construction of maxillary jaw (3 $X$ magnification) using SSD (Shaded Surface Display) showing impacted 25 (encircled) on the right side and open apex of 15 (encircled) on the left side.

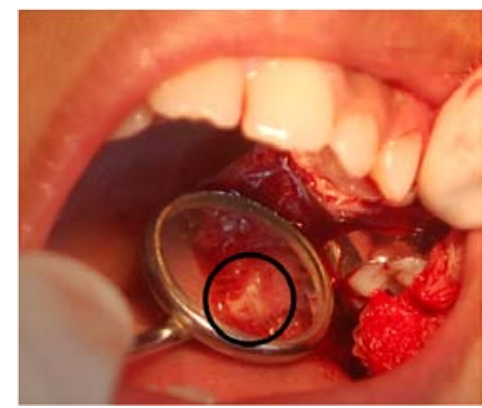

Figure 5: Exposed Crown of Impacted 25

was disimpacted atraumatically with the help of straight elevator in one direction only. The mesio-distal dimension of the tooth was smaller as compared to normal. Maxillary left second premolar had two apices and palatal root was found little dilacerated (Figure 6). These features were not detected in CT scan or OPG. The tooth was tried in the bucco-distal socket of freshly extracted primary maxillary left second molar. As fresh maxillary socket is expandable, the socket was expanded lingually with the help of blunt instrument. The socket was deepened $3 \mathrm{~mm}$ approximately with slow speed drill with the care to preserve periodontal ligaments as much as possible. In the next try maxillary left second premolar was very well accepted by the prepared socket. Its bucco-palatal position was optimum (Figure 7). The tooth was secured in infraocclusion with the help of criss-cross sutures (Figure- maxillary right second premolar). During whole procedure patient remained very co-operative. This positive attitude which can be attributed to parent understands of treatment need and subsequent co- 
Citation: Sharma DS, Singh Chauhan SP, Kishnan R, Sharma S (2012) Autotransplantation of an Impacted Second Premolar in its Predecessor's Socket with the Novel use of Modern Imaging System: A Case Report with 18 Months Follow Up. J Trauma Treat 1:103. doi:10.4172/21671222.1000103

operation and long acquaintance of patient with the author (Dr Divya) with proper motivation and rewards after each extraction previously. Patient was instructed for not brushing the transplanted tooth but to rinse with chlorhexidine mouthwash. Sutures were removed after a week as tooth was found physiologically firm.

After 3 months on recall follow-up tooth was healthy periodontically without any pocket formation or mobility. The tooth was slightly erupted. The tooth responded normally to percussion and electrical pulp testing (EPT). Radiograph of maxillary left second premolar showed regular lamina dura with no signs of ankylosis, replacement resorption or inflammatory resorption (Figure 8). Patient was instructed to contact in case of any adverse symptoms.

After fifteen months of first recall, autotransplanted maxillary left second premolar was found little more erupted and sound periodontically (Figure 9). Premolars on both sides were at same eruption stage (Figure 10). IOPA X-ray showed lengthening of root with apical constriction of maxillary left second premolar (Figure 11) and the tooth responded normally to EPT. Patient was chewing on this tooth with no difficulty. Patient was referred to prosthodontist, who advised for extra-coronal restoration after minor orthodontic mesialization of autotransplanted tooth.

\section{Discussion}

The report presents an unusual case of impaction of maxillary second premolar in an uncommon location. Causes of impaction include crowding, over retained deciduous tooth, trauma, overlying dense bone or soft tissue. No such correlation was found in present case. Early suspicion of impaction of maxillary left second premolar was missed clinically as both maxillary deciduous second molar were

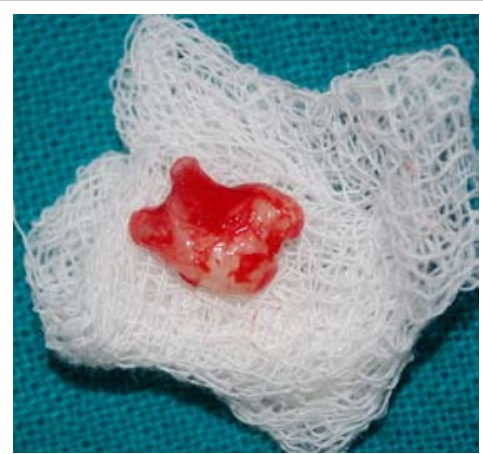

Figure 6: Disimpacted 25 showing bent in Palatal Root and Intact HERS.

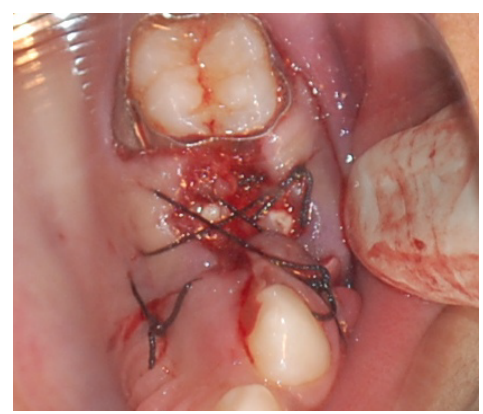

Figure 7: 25, Secured in Socket with criss cross suture.

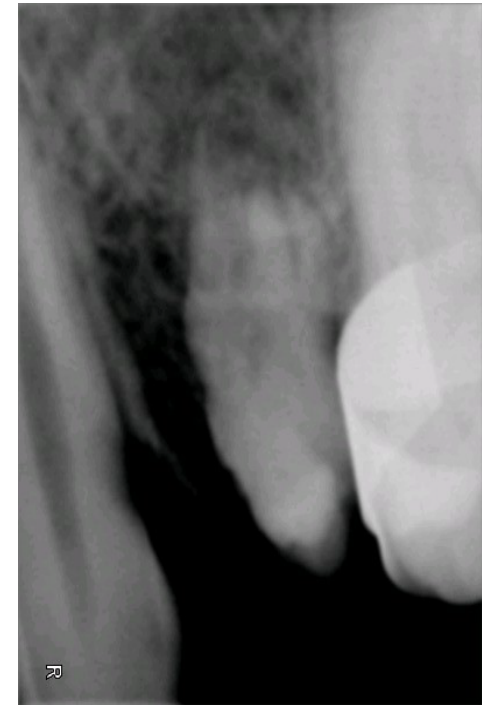

Figure 8: IOPA $X$ ray of autotransplanted 25 after 3 months showing healthy PDL and no pulp pathosis.

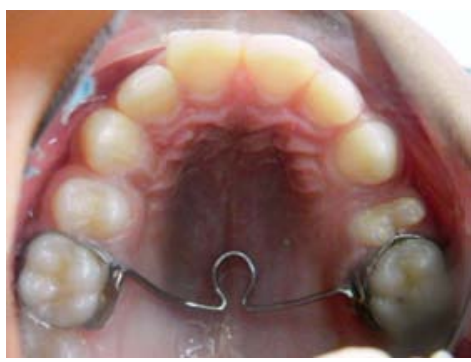

Figure 9: Occlusal view Showing Healthy Gingival Tissues and Erupting 25 after 18 Months of Autotransplantation.

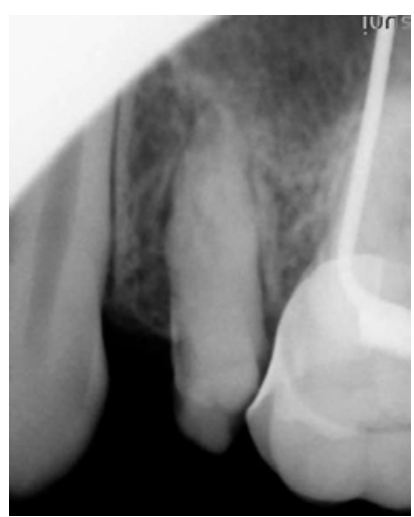

Figure 10: IOPA $X$ ray of autotransplanted 25 showing root lengthening, apical closure and sound bone.

physiologically retained and developing tooth buds of premolars were very small at the time of commencement of serial extraction program.

The latest OPG at the time of visiting our institute showed impacted maxillary left second premolar. CT scan showed that the impaction of second premolar, developing between the distal root of primary maxillary left second molar and mesial root of maxillary left first molar with the crown on palatal side. This unusual position was unidenfiable 
Citation: Sharma DS, Singh Chauhan SP, Kishnan R, Sharma S (2012) Autotransplantation of an Impacted Second Premolar in its Predecessor's Socket with the Novel use of Modern Imaging System: A Case Report with 18 Months Follow Up. J Trauma Treat 1:103. doi:10.4172/21671222.1000103

in OPG (Figure 1). Probably this unusual situation of developing tooth bud resulted in smaller mesio-distal dimension of maxillary left second premolar, though bucco-lingual dimensions were in normal range.

Autotransplantation during mixed dentition period offer advantage of partial root development of premolars. As the disimpacted tooth had stage 2 of root development according to Moorrees [5] (1/2 to 3/4th) clinically and contra-lateral tooth showed open apex in CT scan (Figure 4B), moved us to transplant maxillary left second premolar in its predecessor's socket. As primary maxillary left second molar was non-carious without any rarefaction in surrounding alveolar bone, sound three dimensional bone support was available at recipient site. Sound bone with keratinized mucosa was suggested as necessary for successful autotransplantation [6].

Case studies by Tanaka et al. [7] and Kvint et al. [1] described the autotransplantation of premolars and permanent canines to the recipient site of retained deciduous teeth. Success rate in 4 year follow up was $100 \%$ for premolars and $84 \%$ for canines when recipient sites were in maxilla [7,1]. But autotransplantation of impacted second premolar into its respective predecessor's socket probably, is the first case described here.

Slagsvold and Bjercke8 reported that if integrity of epithelial sheath in the donor tooth was maintained, genetics might control root growth and adequate root formation could be secured. Intact hertwig epithelial sheath on maxillary left second premolar in this case facilitated the root formation and thereby the eruption. The whole surgical procedure i.e. extraction of primary maxillary left second molar, disimpaction of maxillary left second premolar, preparation of socket and autotrasplantation of maxillary left second premolar was completed within 15 minutes by Dr. Divya, thus minimizing the extra-oral time for donor tooth. Tsukiboshi9 found that there was $82 \%$ success rate when premolars were transplanted into the fresh socket while it was $60 \%$ when transplanted to artificially formed socket.

Stage of root formation of donor tooth is an important criteration for the maintenance of vitality, periodontal attachment and full length root completion. Jonsson and Sigurdsson 4 found that 16 out of 40 autotransplanted premolars retained vitality and gained root length upto $11 \mathrm{~mm}$, when root of donor tooth was in stage $2(1 / 2-3 / 4$ th root length, according to Moorrees et al.). In our case donor tooth gained 1:1 crown root ratio after 3 months, which was considered one of the criteria for successful autotransplantation [7,10-12]? Autotransplanted tooth having root length shorter than normal also has been considered as successful [13]. Palatal root of maxillary left second premolar was

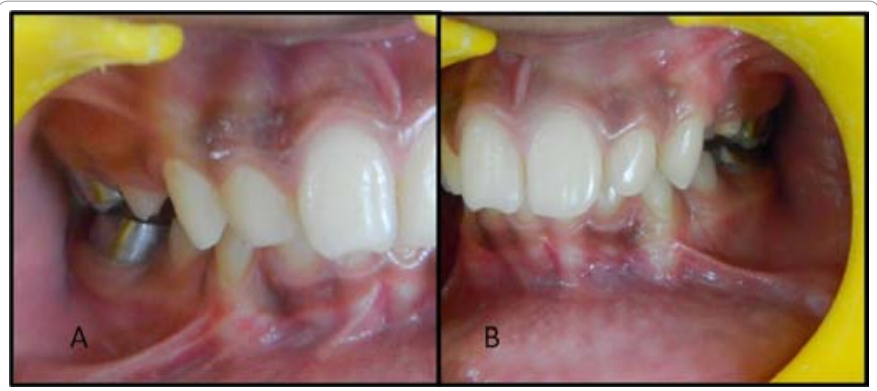

Figure 11: A. Erupting 25, still to reach occlusion. B. Comparable eruption status of 15 . little dilacerated in present case hence root bent was expected Tanaka et al. [7]. Also suggested the need of three dimensional evaluative methods for root development associated with root bending. But even with 3-D CT evaluation root bending of palatal root of maxillary left second premolar could not be made out because of its unusual sandwitched position between maxillary left first molar and primary maxillary left second molar.

At 3 months follow up tooth was seen little erupted while the contra lateral tooth was still retained. This confirms the observation of Paulsen HU et al. [14] that the eruption of immature autotransplanted premolars takes place at a time when periodontal and alveolar healing occurs and before significant root growth is apparent. Contrary to this retarded eruption was found in another study by them [15]. Trauma to HERS while surgery was associated as a possible cause for retarded eruption. In our case HERS was intact. Paulsen HU14 found no tilt in mesial and distal points while eruption of transplanted tooth as those cases were with the straight root. Presence of two root apices combined with bent palatal root made it interesting to observe the transplanted tooth. If both root grow at same rate what would be the resultant vector? None the less after 18 months of transplantation buccal root gained its optimum length with apical closure. No tilt in crown was detected probably because of apical closure before gaining length of palatal root. This might have happened as more resistance was encountered against palatal cortical bone by palatal root and less in maxillary alveolar bone by buccal root.

Serial extraction is started during mixed dentition showing signs of future crowding. Early signs of crowding are usually detected in mandibular anterior region. Permanent tooth buds of second premolar are in their early stage of development at the time of commencement of treatment. This case emphasizes the need of proper evaluation of all the developing permanent tooth buds which may sometimes be missed in routine radiographs. Modern imaging systems like cone beam computed tomography which provide $3 \mathrm{D}$ construction at low cost and reduced radiation should be made mandatory for evaluation of permanent tooth buds for screening of serial extraction cases recently Katheria et al. [16] also have stressed the need of modern imaging systems i.e. cone beam computed tomography not only for early diagnosis but also to know exact location and dimensions of impacted and supernumerary teeth.

\section{Conclusion}

The success of autotransplanted maxillary left second premolar in present case can be attributed to healthy donor tooth with intact periodontal fibers and epithelial sheath, minimal extra oral time for donor tooth, minimum surgical trauma, fresh healthy recipient socket with plenty of left periodontal fibers and the maxilla. Autotransplanted maxillary left second premolar not only served the purpose of preserving alveolar bone three dimensionally but also of chewing functionally. Furthermore autotransplantion eliminated the need for implant in this case as simple extra-coronal restoration will restore its demensions three dimensionally.

\section{References}

1. Kvint S, Lindsten R, M agnusson A, Nilsson P, Bjerklin K (2010) Autotrasplantation of teeth in 215patients. Angle Orthod 80: 446-451.

2. Rao Jahnavi, Fields HW, Chacon GE (2008) Case report: Autotransplantation for a missing permanent maxillary incisor. Pediatr Dent 30: 160-166. 
Citation: Sharma DS, Singh Chauhan SP, Kishnan R, Sharma S (2012) Autotransplantation of an Impacted Second Premolar in its Predecessor's Socket with the Novel use of Modern Imaging System: A Case Report with 18 Months Follow Up. J Trauma Treat 1:103. doi:10.4172/21671222.1000103

Page 5 of 5

3. Aslan BI, Ucuncu N, Dogan A (2010) Long-term follow up of a patient with Long-Term Follow-up of a Patient with Multiple Congenitally Missing Teeth. Angle Orthod 80: 396-404.

4. Jonsson T, Sirgurdsson TJ (2004) Autotransplantation of premolars to premolar site. A long-term follow up study of 40 consecutive patients. Am J Orthod Dentofacial Orthop 125: 668-675.

5. Moorrees CFA, Fanning EA, Hunt EE Jr (1963) Age variation of formation stages for 10 permanent teeth. J Dent Res 42: 1490-1502.

6. Clokie CML, Yau DM, Chano L (2001) Autogenous tooth transplantation: An alternative to dental implant placement? J Can Dent Assoc 67: 92-96.

7. Tanaka T, Deguchi T, Kageyama T, Kanomi R, Inoue M, et al. (2008) Autotransplantation of 28 premolar donar teeth in 24 orthodontic patients. Angle Orthod 78: 12-19.

8. Slagsvold O, Bjerck B (1974) Autotransplantation of premolars with partly formed roots. Am J Orthod 66: 355-366.

9. Tsukiboshi M (2002) Autotransplantation of teeth: predictable success. Dent Traumatol 18: 157-180.

10. Schwarz O, Bergmann P, Klausen B (1985) Resorption of autotransplanted human teeth: a retrospective study of 291 transplantation over a period of maxillary left second premolar years. Int Endon J 18: 119-131.

11. Kristerson L, Lagerstrom $L$ (1991) Autotransplantation of teeth in cases with agenesis or traumatic loss of maxillary incisors. Eur J Othod 13: 486-492.

12. Kugelberg R, Tegsjo U, Malmgren O (1994) Autotransplantation of 45 teeth to the upper incisor region in adolescents. Swed Dent J 18: 165-172.

13. Czochrowska EM, Stenvik A, Bjerke B, Zachrisson BU (2002) Outcome of tooth transplantation: survival and success rates $17-41$ years posttreatment. Am J Orthod Dentofacial Orthop 121: 110-119.

14. Paulsen HU, Shi X-Q, Welander U, Huggare J, Scheutz F (2001) Eruption pattern of transplanted premolars visualized by radiographic color coding. Am J Orthod Dentofacial Orthop 119: 338-345.

15. Paulsen HU, Andreason JO, Schwartz O (1995) Pulp and periodontal healing root development and root resorption subsequent to transplantation and orthodontic rotation: a long term study of autotransplanted premolars. Am J Orthod Dentofacial Orthop 108: 630-640.

16. Katheria BC, Kau CH, Robert T, Jung-Wei C, Jeryl E, et al. (2010) Effectiveness of Impacted and Supernumerary Tooth Diagnosis from Traditional Radiography Versus Cone Beam Computed Tomography. Pediatr Dent 32: 304-309. 